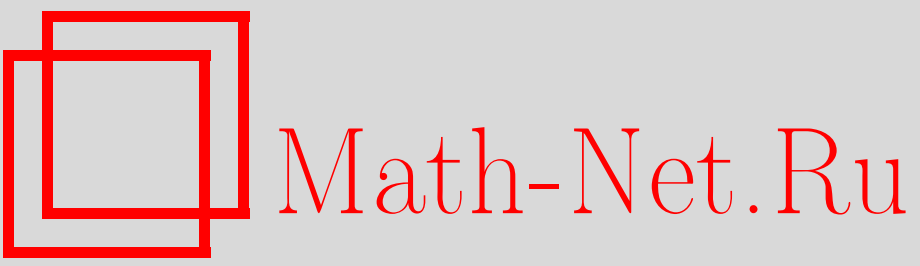

Л. Д. Пустыльников, Вероятностные законы в распределении дробных частей значений многочленов и обоснование гипотезы квантового хаоса, УМH, 1999, том 54, выпуск 6, 173-174

DOI: https://doi.org/10.4213/rm244

Использование Общероссийского математического портала Math-Net.Ru подразумевает, что вы прочитали и согласны с пользовательским соглашением

http://www . mathnet.ru/rus/agreement

Параметры загрузки:

IP: 54.197 .130 .99

26 апреля 2023 г., 12:22:27 


\title{
ВЕРОЯТНОСТНЫЕ ЗАКОНЫ В РАСПРЕДЕЛЕНИИ ДРОБНЫХ ЧАСТЕЙ ЗНАЧЕНИЙ МНОГОЧЛЕНОВ И ОБОСНОВАНИЕ ГИПОТЕЗЫ КВАНТОВОГО ХАОСА
}

\author{
Л. Д. Пустыльников
}

Эта работа посвящена классической задаче о распределении дробных частей значений многочленов, которая, начиная с работ Харди, Литтлвуда и Г. Вейля [1], [2], привлекает к себе большое внимание. Данную работу стимулировали следующие два обстоятельства: 1) давно был проявлен интерес к доказательству центральной предельной теоремы для распределения дробных частей значений различных функций, и, в частности, она была доказана для показательных функций определенного вида [3] , [4]; 2) исследование распределения расстояний между соседними дробными частями значений многочленов вызвано проблемой обоснования гипотезы квантового хаоса, сами эти расстояния соответствуют расстояниям между соседними энергетическими уровнями некоторых квантовых систем, а гипотеза состоит в том, что закон распределения этих расстояний в общем случае квазипуассоновский, т.е. имеет плотность $\rho(\sigma)$, близкую к $\rho(\sigma)=e^{-\sigma}[5]-[7]$.

Основные результаты работы - это усиленный закон больших чисел и центральная предельная теорема в распределении дробных частей от сумм значений бесконечного числа многочленов с попарно различными степенями (теорема 1), предельные законы распределения дробных частей и расстояний между ними для фиксированного многочлена (теорема 2) и обоснование сформулированной выше гипотезы (теорема 3 и ее следствие), причем в отличие от первых двух теорем приведено доказательство теоремы 3 , которое есть следствие одной общей теоремы из работы [8].

В случае многочленов произвольных степеней, рассматриваемых в теореме 1 , вероятностное пространство есть бесконечномерный тор $T=\left\{a=\left(a_{0}, a_{1}, \ldots\right): 0 \leqslant a_{s}<1(s=0,1, \ldots)\right\}$, являющийся произведением счетного числа единичных окружностей $0 \leqslant a_{s}<1(s=0,1, \ldots)$, вероятность $\mathrm{P}$ есть мера на $T$, являющаяся прямым произведением обычных длин на этих окружностях, а дробная часть $\left\{f_{n}(x)\right\}$ значения многочлена $f_{n}(x)=a_{0}+a_{1} x+\cdots+$ $a_{n} x^{n}$ степени $n$ в точке $x$ есть случайная величина от случайно выбранной точки $a=$ $\left(a_{0}, a_{1}, \ldots, a_{n}, a_{n+1}, \ldots\right) \in T$. То же самое касается дробной части $\left\{\sum_{\nu=s_{1}}^{s_{2}} f_{n}(\nu)\right\}$ от суммы значений многочлена $f_{n}(x)$ в последовательных точках $\nu$, которая также является случайной величиной, зависящей от точки $a=\left(a_{0}, a_{1}, \ldots\right) \in T$, на вероятностном пространстве $(T, \mathrm{P})$.

Теорема 1. Рассмотрим последовательность случайных величин $\zeta_{k}=\zeta_{k}(a)(k=$ $1,2, \ldots)$ на вероятностном пространстве $(T, \mathrm{P})$ таких, что $\zeta_{k}(a)=\left\{\sum_{\nu=s_{1 k}}^{s_{2 k}} f_{n_{k}}(\nu)\right\}$ есть дробная часть суммы значений многочлена $f_{n_{k}}(x)=a_{0}+a_{1} x+\cdots+a_{n_{k}} x^{n_{k}}$ степени $n_{k} \geqslant 1$ в натуральных числах $\nu$, расположенных на отрезке $s_{1 k} \leqslant \nu \leqslant s_{2 k}$. Предположим, что все числа $n_{k}$ попарно различны между собой. Тогда справедливы следующие утверждения:

1. с вероятностью 1

$$
\lim _{N \rightarrow \infty} \frac{1}{N} \sum_{k=1}^{N} \zeta_{k}=\frac{1}{2} ;
$$

2. при $N \rightarrow \infty$ распределения вероятностей случайньх величин

$$
\kappa_{N}=\frac{1}{\sqrt{N / 12}}\left(\sum_{k=1}^{N} \zeta_{k}-\frac{N}{2}\right)
$$

слабо сходятся $к$ гауссовскому нормальному распределению с плотностью

$$
\frac{1}{\sqrt{2 \pi}} \exp \left(-\frac{x^{2}}{2}\right)
$$


Теорема 2. Предположим, что у многочлена $f_{n}(x)=a_{0}+a_{1} x+\cdots+a_{n} x^{n}$ степени $n \geqslant 1$ среди коэффициентов $a_{1}, \ldots, a_{n}$ есть иррациональное число. Тогда справедливь равенства

$$
\lim _{N \rightarrow \infty} \frac{1}{N} \sum_{k=1}^{N}\left\{f_{n}(k)\right\}=\frac{1}{2}, \quad \lim _{N \rightarrow \infty} \frac{1}{N} \sum_{k=1}^{N}\left|\left\{f_{n}(k+1)\right\}-\left\{f_{n}(k)\right\}\right|=\frac{1}{3} .
$$

ТЕОРема 3. Предположим, что у многочлена $f_{n}(x)=a_{0}+a_{1} x+\cdots+a_{n} x^{n}$ степени $n \geqslant 2$ среди коэффичиентов $a_{2}, \ldots, a_{n}$ есть иррациональное число; $\sigma$ - прочзвольное число из интервала $0<\sigma \leqslant 1 ; N$ - натуральное число; $D_{N}(\sigma)$ - число чисел в ряду $k=1, \ldots, N$ maких, что $0 \leqslant\left\{f_{n}(k+1)\right\}-\left\{f_{n}(k)\right\}<\sigma$. Тогда

$$
\lim _{N \rightarrow \infty} \frac{1}{N} D_{N}(\sigma)=\sigma-\frac{\sigma^{2}}{2} \text {. }
$$

ДоказАТЕЛЬСтво. Рассмотрим плоский двумерный тор $T_{2}=\{x, y: 0 \leqslant x<1,0 \leqslant y<1\}$ и функцию $\chi(x, y)$ на нем, имеющую вид

Тогда имеем:

$$
\chi(x, y)= \begin{cases}1, & \text { если } 0 \leqslant y-x<\sigma \\ 0 & \text { в противном случае. }\end{cases}
$$

$$
\frac{1}{N} D_{N}(\sigma)=\frac{1}{N} \sum_{k=1}^{N} \chi(\{f(k)\},\{f(k+1)\}),
$$

и утверждение теоремы 3 сводится к доказательству того, что при $N \rightarrow \infty$ правая часть последнего равенства сходится к интегралу

$$
\iint_{T_{2}} \chi(x, y) d x d y=\sigma-\frac{\sigma^{2}}{2}
$$

Но этот факт следует из теоремы I, доказанной в $\S 3$ гл. II работы [8]. Теорема 3 доказана.

СледСтвиЕ. Если $D_{N}(\sigma)$ - величина, введенная в формулировке теоремы 3 , то предельная функция распределения $P(\sigma)=\lim _{N \rightarrow \infty} \frac{1}{N} D_{N}(\sigma)$ отличается от функции пуассоновского распределения, имеющего плотность $\rho(\sigma)=\exp (-\sigma)$, на величину третьего порядка малости по $\sigma$ в области $0 \leqslant \sigma \leqslant 1$.

ДокАЗАТЕльСтво. Функция пуассоновского распределения есть интеграл

$$
\int_{0}^{\sigma} e^{-s} d s=1-e^{-\sigma}=\sigma-\frac{\sigma^{2}}{2}+O\left(\sigma^{3}\right),
$$

и утверждение следствия вытекает из теоремы 3.

Выражаю глубокую благодарность F. Götze, заинтересовавшему меня указанными проблемами, Т.В. Локоть и Г.А. Маргулису за полезные обсуждения, a Sonderforschungsbereich 343 университета г. Билефельд-за поддержку.

\section{СПИСОК ЛИТЕРАТУРЫ}

[1] Hardi G.H., Littlewood J. E. // Proc. Intenat. Congr. Math. Cambridge. 1912. P. 223-229. [2] Weyl H. // Math. Ann. 1916. V. 77. P. 313-352. [3] Kac M. // Ann. of Math. (2). 1946. V. 47. №1. P. 33-49. [4] Fortet R. // Studia Math. 1940. V. 1. P. 54-69. [5] Berry M. V., Tabor M. // Proc. Roy. Soc. London. Ser. A. 1977. V. 356. P. 375-394. [6] Casati G., Chirikov B. V., Guarneri I. // Phys. Rev. Lett. 1985. V. 54. P. 1350-1353. [7] Knauf A., Sinai Ya. G. Classical Nonintegrability, Quantum Chaos. Basel: Birkhäuser, 1997. (DMV Seminar. V. 27.) [8] Пустыльников Л. Д. // УМН. 1993. Т. 48. № 4. С. 131-166. 